simply that too little is known of how the brain is constructed, and how it becomes what it is, for the analogy to be fruitful.

Indeed, the attempts that have been made to build models of single neurons, so as to represent their electrical response in terms of the external influences to which they are subjected, yield only poor results. There are simply too many channels of influence, electrical and otherwise, on the surface of a neuron, for its behaviour to be modelled in other than a symbolic fashion in the present state of observation.

The question remains of how the human brain becomes what it is. Observations with newborn mammals have shown that even the visual system remains plastic for several weeks after birth, presumably because neuronal axons have to learn to find their way to appropriate partners. That, fortunately, is a process that can be studied directly.

For people, the practical question will be to form a general view of how long the various functions of the brain remain susceptible to learned influences. Among other things, that may help to set psychology, for too long a Cinderella science, on a firm foundation for the first time in history.

\section{Promise of biodiversity}

\author{
IMAGE \\ UNAVAILABLE \\ FOR COPYRIGHT \\ REASONS
}

Rainforest in Queensland, Australia, typical of the terrain protected by the Biodiversity Convention signed at Rio de Janeiro in August 1992 (but already safeguarded by the Australian federal government). Devising ways of estimating biodiversity quantitatively remains an unsolved problem.

\section{Energy source for cells}

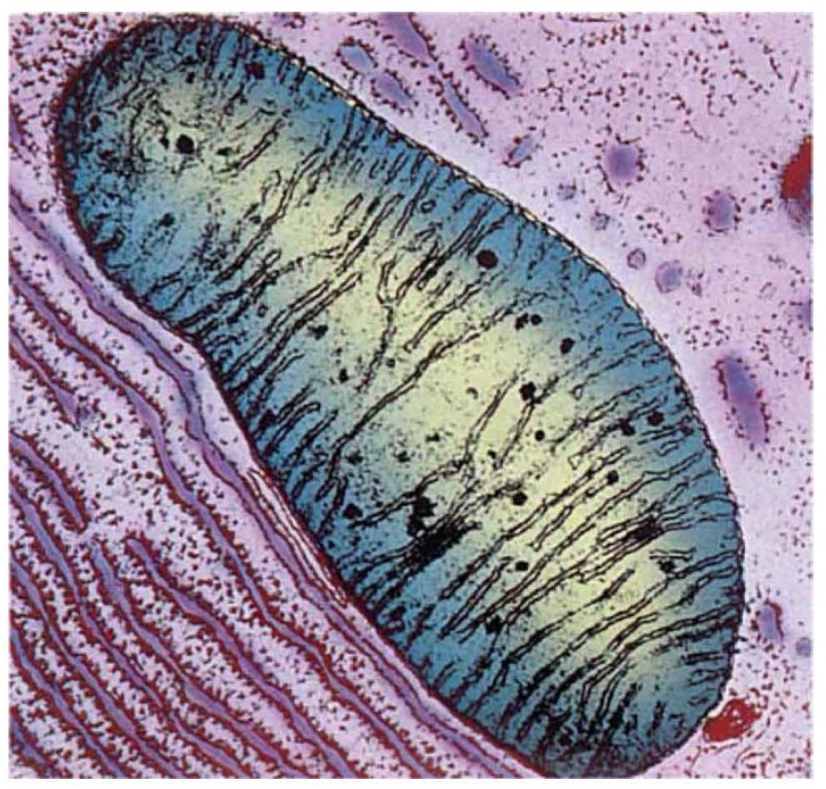

Electron micrograph of a single mitochondrion from a human pancreas cell, showing the cristae (internal folds of the inner membrane) at which respiration occurs. Also visible is the endoplasmic reticulum within the mitochondrion, carrying bead-like ribosomes (for protein synthesis). Mitochondria have their own complement of DNA, responsible for the production of some mitochondrial proteins, which replicates independently of the nuclear DNA. Mitochondria are believed to have derived from Archaean symbiotic bacteria. (Science Photo Library)

\title{
Molecules in embryology
}

THERE is a well-known gene in the fruit-fly Drosophila called eyeless, whose mutants have no eyes. In the mouse, there is a similar ('homologous') gene called smalleye, whose mutants have small eyes. Yet the fly's eye is a compound eye and the mouse eye an integral structure like that of other vertebrates.

So much was reported at the annual meeting of drosophilists at Crete this year by Walter Gehring, of the Biozentrum at Basel, the one who discovered more than a decade ago the genetic hallmark of the class of genes known as homeotic genes - a short sequence of DNA upstream of the gene proper and now known as the "homeobox".

This unexpected finding is in itself a curiosity. But it is a vivid sign that the development from embryo to adult of two very different organisms may have something substantial in common with each other. If nothing else, this justifies the long-standing use of Drosophila as the work-horse of the study of development.

In reality, two kinds of study are intertwined. Development is another name for embryology, which includes the metamorphoses that insects undergo. But embryonic development leads to the specialization of cell types, and thus to the silencing of inappropriate genes (by a mechanism not yet understood). There are two switches to find: on and off.

Molecular techniques have enormously helped to transform both fields in the past few years. The search now is for the diffusion of gene products of vertebrate homeotic genes that play parts analogous to those of the gene products of the corresponding genes in Drosophila, which appear by their movement through the body of the developing embryo to regulate the specialization of cells. This is a door that will need little pushing to swing open. 